\title{
Opportunities for collaboration between taxonomists and geneticists to database curation
}

\author{
Mikel Aguirre ${ }^{\ddagger}$, Joana Hevia-Orube ${ }^{\ddagger}$, Laura Ocabo ${ }^{\ddagger}$, Jesús Arrate§, Eva López§ , Jose Manuel \\ Leonardo§, Alberto Aguirre§, Begoña Gartzia de Bikuña ${ }^{\ddagger}$ \\ $\ddagger$ Anbiotek Biotechnologies - Anbiolab, Derio, Spain \\ $\S$ Anbiotek, Erandio, Spain
}

Corresponding author: Mikel Aguirre (magirre@anbiolab.com), Joana Hevia-Orube (jhevia@anbiolab.com)

Received: 25 Feb 2021 | Published: 04 Mar 2021

Citation: Aguirre M, Hevia-Orube J, Ocabo L, Arrate J, López E, Leonardo JM, Aguirre A, Gartzia de Bikuña B

(2021) Opportunities for collaboration between taxonomists and geneticists to database curation. ARPHA

Conference Abstracts 4: e65051. https://doi.org/10.3897/aca.4.e65051

\begin{abstract}
Ecological status class assessments of European rivers are required by the EU Water Framework Directive (WFD), the purpose of which is to carry out good river basin management. In the future, these studies will be presumably complemented by molecular analysis, additional to classical taxonomy. To ease this transitional phase, several aspects must be addressed.
\end{abstract}

One of the limitations of ecological community studies based on massive sequencing techniques is the quality of reference databases. There are usually two major problems to deal with: 1) the absence of reference sequences for certain organisms and 2) the presence of sequences of doubtful quality or incorrect assignment. To prevent this type of problem, good coordination between qualified taxonomists and genetic analysts is necessary. Taking into account that we are a group made up of expert taxonomists and bioinformaticians, we have carried out a review of several species. Specifically, we have focused on organisms of interest present in the hydrological network for monitoring the ecological state of waters of the autonomous community of the Basque Country.

In this review, a historical study of the species present throughout the entire period of the monitoring hydrological network (30 years) was first carried out. Then a search was carried out in the genetic databases to see how many of these species have not a reference 
sequence. Thus be able to start a search and selection for said organisms by a taxonomist and to sequence them.

In parallel, a study of the existing sequences in the databases was carried out to confirm the clustering of the sequences at the family level. In the case of the study of macroinvertebrates, we test a selected gene or regions (18S V4 rDNA, 18S V9 rDNA, $\mathrm{COI})$. to check their variability. As follows, it was possible to discard sequences that were significantly dispersed from the family cluster and to prevent sequences that could be incorrectly assigned to each family. Furthermore, it was observed that for the macroinvertebrates of interest for our geographical area, a total of 154 families, 27 $(17.53 \%)$ were missing for having reference sequences.

In the case of the sequences of the freshwater fish population, we discovered the following situation: of 34 species with occurrences in the rivers of the network, 16 had no reference sequences. Therefore, taking advantage of the annual samplings of the hydrological network, samples of these fish species were collected to be able to include their sequences in the database. Once the reference sequences of the species were obtained, a macroinvertebrate and ichthyofauna detection project was carried out. In this project, we use amplicon technology by Illumina sequencing focused focused on eDNA from water samples. In which it was possible to observe the improvement of detection and species assignment once the sequences were entered into the database.

In conclusion, getting a complete and curated database is essential for the correct assignment and detection of organisms to improving the accuracy analysis and interpretation of the communities. In the same way, the need for collaboration between taxonomists and geneticists is imperative to be able to correctly validate the reference sequences.

\section{Keywords}

$18 \mathrm{~S}$ rDNA, COI, macroinvertebrates, fish, curated databases

\section{Presenting author}

Mikel Aguirre

\section{Presented at}

1st DNAQUA International Conference (March 9-11, 2021) 


\section{Acknowledgements}

Marta Rozas Ormazabal, Departamento de Biodiversidad del Gobierno Vasco.

Alberto Manzanos Arnaiz, Uraren Euskal Agentzia.

\section{Conflicts of interest}

No conflicts of interest. 